\title{
Referentes culturales en la enseñanza de español como lengua extranjera (ELE) en Chile
}

\author{
Cultural references in the teaching of Spanish as a foreign language \\ (ELE) in Chile
}

\author{
Gloria Macarena Toledo Vega \\ María García Fernández ${ }^{2}$ (1) @ \\ ${ }^{1}$ Pontificia Universidad Católica de Chile (UC); ${ }^{2}$ Universidad de Granada (UGR), España.
}

\section{Resumen}

En la enseñanza del español como segunda lengua o lengua extranjera es considerable el valor de la adquisición de los conocimientos culturales en el desarrollo de la competencia intercultural. Tradicionalmente la formulación y la descripción de los contenidos culturales en ELE se ha elaborado a partir de patrones generalistas. En este estudio se analizan los intereses y motivaciones de los alumnos de la Pontificia Universidad Católica de Chile (UC), para observar coinciden con los referentes culturales de los descriptores de los manuales de ELE. La investigación plantea una evaluación de los contenidos culturales de ELE en Chile y una reformulación de su enseñanza para el desarrollo de un aprendizaje significativo, pues se observa una a falta de correspondencia entre los referentes culturales seleccionados por los estudiantes y aquellos que tradicionalmente están presentes en los manuales de ELE.

Palabras clave: aprendizaje de la cultura; español como lengua extranjera; conocimiento cultural; español como lengua extranjera.

\begin{abstract}
In the teaching of Spanish as a second or foreign language, the value of acquiring cultural knowledge in the development of intercultural competence is considerable. Traditionally the formulation and description of cultural contents in ELE has been developed from generalist patterns. This study analyzes the interests and motivations of the students of the Pontifical Catholic University of Chile to observe if they coincide with the cultural references as the descriptors of the Spanish as a foreign language manual.The research proposes an evaluation of ELE's cultural content in Chile and a reformulation of its teaching for the development of meaningful learning, because a lack of correspondence between cultural references selected by students, and those in SFL texts it is observed. Keywords: cultural learning; spanish as a foreign language; cultural knowledge.
\end{abstract}

\section{Introducción}

En la actualidad es sabido que la adquisición de una lengua extranjera se potencia al trabajar la competencia cultural en el aula (Carcedo, 1998; De Santiago, 2010). Conscientes de esto, los docentes se han preocupado de incorporar referentes culturales en sus clases de lengua extranjera o segunda lengua (LE/SL). Sin embargo, la selección de los aspectos culturales que se deben enseñar en el aula no es una

Recomendación de cómo citar este artículo (APA 6)

Toledo, G. M. y Fernández, M. G. (2020). Referentes culturales en la enseñanza de español como lengua extranjera (ELE) en Chile. Revista Iberoamericana de Educación, 82(2),107-130. https://doi.org/10.35362/rie8223566 
tarea fácil. En el desarrollo de la enseñanza-aprendizaje de español como lengua extranjera (ELE), se ha organizado la cultura tomando como referencia parámetros generalistas de diversos campos: la economía, la música, la literatura, la historia, entre otros.

Un tratamiento efectivo de la cultura necesita la detección de patrones culturales para conocer si los intereses de los alumnos coinciden con los contenidos que presentan los manuales de español. Tomando en cuenta la enorme variedad de dialectos del español en el mundo hispanoamericano, esbozar una realidad cultural en un manual de ELE no es sencillo. Este hecho puede suceder si se toma en cuenta que la mayoría de los manuales de ELE son publicados sobretodo en España o en Estados Unidos.

Para investigar la forma en que se representa una cultura en la enseñanza de ELE, centramos nuestra investigación en Chile, país en que investigaciones como las de Ferreira (2017) revelan la ausencia de materiales didácticos que presenten el español de esta localidad. En efecto, solamente desde el año 2017 existe el manual Punto C/ELE (Toledo, 2017; Toledo, 2018; Bravo, 2018) como único representante de la cultura y variedad chilena del español. En esta situación cobra importancia la investigación del desarrollo de la competencia intercultural en el área de la enseñanza del español en Chile, como una forma de evidenciar la necesidad de emplear materiales comunicativos que representen la diversidad lingüística-cultural de dicha comunidad específica, con el objeto de que el alumno de inmersión reconozca la cultura meta de primera fuente en sus materiales de ELE. El conocimiento de los patrones culturales en la enseñanza de ELE en Chile ayudará a conocer el panorama actual de la enseñanza de español en las aulas chilenas y permitirá establecer el perfil del alumno extranjero que decide estudiar español en este país.

En este trabajo, el contenido cultural se investigará en encuestas de medición organizadas en dos ejes: el nivel de frecuencia de los referentes culturales y el nivel de motivación sobre dichos ítems. Los datos obtenidos de 51 informantes estudiantes de intercambio en la UC, ayudarán a la actualización de los patrones culturales enseñables en ELE, según las nociones de los mismos aprendientes de español durante su aprendizaje. 


\section{Tendencias en la enseñanza del componente cultural en lenguas extranjeras}

Previo al apogeo del enfoque comunicativo, el tratamiento de la cultura, desde una visión estereotipada y descontextualizada, redundaba en una práctica que desconectaba los objetivos de lengua de los elementos culturales. Esta situación puede persistir en la actualidad, si tomamos en cuenta que la mayoría de los manuales de LE son diseñados y publicados en unos pocos países del mundo. Al respecto, señala que los aprendientes comparten una orientación negativa hacia los textos de enseñanza, pues consideran que la información que entregan es limitada, deja poco espacio a los temas pragmáticos y no provee información ni práctica suficiente para lograr un comportamiento "natural" en la lengua.

En los más recientes manuales de LE, Derenowski (2011) detecta tendencias positivas en la enseñanza de la cultura, que implican la promoción de actividades interculturales; el tratamiento de temas sociales serios; material de presentación auténtico y la promoción de un intercambio de perspectivas. Pese a lo anterior, también se observan tendencias negativas: un foco excesivo en la forma; una orientación preferente hacia el turismo -para evitar el tratamiento de temas más controvertidos- y la presentación estereotipada de la cultura meta y la cultura propia de los estudiantes. A estas tendencias podríamos sumar aquella que trata la cultura con un foco en la comparación (Lee, 2015) que suele dejar en ventaja a un componente por sobre el otro.

Nuestra perspectiva en relación con la enseñanza de la cultura en LE/SL comparte la idea de Areizaga (2001) respecto a promover un cambio de orientación en la enseñanza de la cultura, cuyo énfasis debe estar en desarrollar la capacidad para comprender y comunicarse con otros, es decir, en el fomento de la interculturalidad,

\section{Hacia una definición de la cultura en la enseñanza de lenguas extranjeras}

En el campo de la enseñanza de LE, la cultura presenta diferentes definiciones según los autores que la investigan. García (2004) plantea las variables imprescindibles que, de acuerdo con esta autora, determinan la cultura: características medio ambientales; condiciones demográficas; parámetros de conducta asociados con ceremonias y creencias; convenciones sociales; nivel de desarrollo social y tecnológico de las diversas sociedades; contactos corporales; hábitos diarios; literatura; tradiciones; 
salud; educación; gestos; hogar y humor. A estos elementos, Carcedo (1998) agrega la idea que se forman los hablantes de las lenguas sobre culturas extranjeras. El Grupo CRIT (2006) (Comunicación y Relaciones Interculturales y Transculturales, que estudia de las interacciones comunicativas entre inmigrantes y naturales de la Comunidad Valenciana), toma en cuenta elementos similares, poniendo énfasis en las dimensiones comunicativas extralingüísticas, dado que de estas tratan en general los estudios de comunicación intercultural. Díaz (2006) resume los elementos que se han señalado y afirma que nuestro ambiente en sí es cultural, ya que todos ellos podemos aprender modos para comunicarnos, consciente o inconscientemente, puesto que la cultura se conforma a partir de un comportamiento aprendido y adquirido de momento en que el individuo está inmerso en una sociedad (García, 2004).

El Diccionario de Términos Clave (Instituto Cervantes, 2009) hace referencia a la cultura en relación más directa con componentes de la lengua. Según este, la cultura implica elementos léxicos para los que no hay equivalente semántico en la propia lengua; elementos léxicos cuyo sentido corre el riesgo de ser transferido erróneamente al propio contexto sociocultural y medios no verbales de expresión, como formas de saludo o despedida, o los usos convencionales de la lengua en comportamientos rituales cotidianos, por ejemplo, formas de invitar, de aceptar o declinar invitaciones. El mismo texto indica lo que se debe conocer en cuanto a referentes culturales españoles:

a) Conocimientos generales sobre los países hispanos.

b) Saberes y comportamientos socioculturales.

c) Habilidades y actitudes interculturales para la configuración de una identidad plural que implica la mediación cultural.

Ahora bien, las distintas variables que forman parte del concepto de cultura pueden clasificarse en distintos ámbitos. Navarro (2009) identifica tres tipos de cultura que denomina "cultura enciclopédica, epidérmica y pragmática" y que define del siguiente modo:

a) Cultura enciclopédica: en el terreno de lo consciente y estudiado.

b) Cultura epidérmica: en el terreno de lo inconsciente. Se trata de pautas de comportamiento que se desarrollan de forma paralela al proceso de adquisición de la lengua materna.

c) Cultura lingüística o pragmática. Corresponde a la forma en que se expresa una comunidad lingüística y cultural (Navarro, 2009). 
Cualquiera sea el conjunto de variables hemos de considerar que no podemos hablar de una cultura estática; la cultura siempre está en movimiento y en contacto con otras culturas: "está fuertemente marcada por elementos como, los fenómenos de la moda, los cambios debidos a evoluciones tecnológicas y técnicas, la diversidad de ideologías, las aportaciones de otras culturas, etc." (Denis y Matas 2009, p. 88). Nuestro estudio, recoge la idea de una cultura en movimiento y por eso se preocupa de recoger el interés actual de los estudiantes de ELE en una comunidad cultural específica: la chilena.

\section{Enseñar la cultura local en contexto de inmersión}

La enseñanza en contexto de inmersión es una oportunidad para que los aprendientes de una segunda lengua se familiaricen con la cultura meta experimentándola en el diario vivir. Para esto, sin embargo, es necesario contar con una especie de guía cultural que ayude a entender las costumbres, procesos y dinámicas de la sociedad meta. En este sentido, parece muy relevante que la enseñanza de ELE en contexto de inmersión enfatice en la enseñanza de la cultura local, más aún considerando la diversidad de culturas que comprende la cultura hispánica en general.

Como se desprende del apartado anterior, la cultura abarca, con una considerable amplitud, el contexto global de una comunidad de habla. La atención a las características del contexto en la comunicación se resume en el concepto de competencia comunicativa desarrollado por Hymes (1972), el cual vincula el dominio de la gramática a una serie de destrezas que la complementan. Entre estas destrezas el componente social (Canale y Swain, 1980) ocupa un lugar fundamental, porque este nos ayudará a entender muchos de los comportamientos comunicativos y a desenvolvernos de forma adecuada de acuerdo con las convenciones que rigen el lenguaje en una comunidad de hablantes (Quiles y Cabrera, 2013). En palabras de Denis y Matas (2009) la enseñanza - aprendizaje del componente cultural "significa desarrollar en el aprendiz capacidades a nivel pragmático para que pueda actuar de manera adecuada, pero también capacidades que le permitan "administrar" su aprendizaje: es decir, ser consciente de las distintas herramientas cognitivas de las que dispone para aprender" (p. 87).

La escasez de material de ELE en Chile podría explicar la dificultad para encontrar investigaciones sobre los parámetros culturales de esta comunidad de habla, aplicados a la enseñanza del español. A su vez, esta escasez responde a la relativa corta data en la aparición de los primeros centros de ELE (años 90); a que en este país la 
enseñanza de ELE es impartida por escuelas que no forman parte de ninguna red oficial; y a que solo desde el 2012 al presente se generaliza la enseñanza de ELE en el ámbito universitario, con avances en formación de profesores, la asociación de instituciones universitarias al Instituto Cervantes e incluso avances en la certificación de ELE (Ferreira, 2017; Quirós, 2018).

\section{Implicancias de la enseñanza de la cultura en una lengua extranjera}

De acuerdo con un estudio de Viñó y Massó (2015), la enseñanza de la cultura en LE plantea cierta dificultad para los profesores, en el sentido de encontrar temas que interesen a todos, evitando los estereotipos y la generación de situaciones incómodas al tratar temas idiosincráticos. Esto porque lo que en una cultura se puede percibir como algo natural, en otra podría ser condenado; por ejemplo, el uso cariñoso de adjetivos como gordo/a; negro/a o viejo/a entre parejas chilenas, es percibido como una falta de respeto por aprendientes estadounidenses o europeos.

Enseñar el contenido cultural en una lengua extranjera implica tomar decisiones sobre perspectivas: qué enseñar y qué cultura, intentando evitar los estereotipos, o bien propiciando una reflexión sobre los orígenes, implicaciones y posibles cambios de estos. Dado que la cultura no es estática y que los intereses de nuestros aprendientes pueden diferir mucho según la edad, la ocupación o la cultura materna, los análisis de necesidades o investigaciones pueden ser de gran utilidad. Otwinowska-Kasztelanic (2011) contrasta perspectivas de dos grupos etarios y concluye que los contenidos asociados a la cultura pueden variar según la edad de los aprendientes; los adolescentes evidencian una motivación más instrumental que integrativa, y en general, se interesan más bien por datos aislados sobre la cultura meta. Los profesores de inglés como lengua extranjera, por su parte, no parecen muy conscientes sobre lo que sus estudiantes quieren aprender y focalizan la enseñanza de la cultura en el Reino Unido y los EE. UU., en lugar de aportar una perspectiva que aborde otras culturas menos conocidas.

La evaluación de la competencia cultural es otro desafío para el profesor, quien tiene que aprender cómo enseñar dentro de un contexto cultural. En palabras de Corros, "la tarea no es enseñar simplemente a decir cosas, sino guiar y ayudar a los estudiantes en el descubrimiento de que el sistema de una nueva lengua conduce a nuevas formas de percepción, de clasificación y de categorización, a nuevas formas de interactuar, de ver y de conocer el mundo." (Corros, 2008, p. 2). En un intento para que los aprendientes manifiesten sus inquietudes respecto al aprendizaje de la 
cultura meta, Corros propone la creación de un diario de aprendizaje como recurso didáctico y personal en el que el alumno puede escribir sus experiencias tanto dentro como fuera del aula. Este diario ofrece al alumno la oportunidad de expresar sus opiniones sobre aspectos implicados en su proceso de adaptación cultural y de asimilación de elementos. Por su parte, el profesor de ELE puede utilizar este recurso como una fuente de información sobre los aprendientes, especialmente en aspectos que son relevantes en la adquisición de la competencia oral (de carácter sociocultural, cognitivo o estratégico) y que pocas veces son evaluados por los profesores de LE.

Los desafíos para los docentes de LE que enseñan cultura van desde conocer el nombre de sus alumnos y el significado que estos nombres pueden tener en sus respectivas culturas (ver Akinyemi, 2011 para el caso de los nombres en yoruba y el contenido cultural de estos) hasta comprender qué conductas y normas culturales son más fáciles y más complejas de adquirir entre los aprendientes de distintas culturas de origen.

\section{Razones para la enseñanza - aprendizaje de la cultura en LE}

En el plano lingüístico, Carcedo (1998) considera la cultura como una competencia que aumenta y enriquece los conocimientos de la lengua aprendida por parte del estudiante. En este sentido, estamos de acuerdo con la observación de Sapir (1954) respecto a que "es una ilusión pensar que podamos comprender los rasgos salientes y significativos de una cultura simplemente a través de la observación y sin la dirección del simbolismo lingüístico que hace que estos rasgos sean significativos e inteligibles para la sociedad" (p. 161).

En el plano cognitivo, la cultura puede apoyar el desarrollo del pensamiento crítico ya que "el alumno contrasta la nueva cultura con la propia, cuestiona la información y analiza las diferencias" (Níkleva 2012 , p. 167). En términos de Byram y Fleming, "la competencia intercultural implica un cuestionamiento -implícito y, a veces, explícito- de las suposiciones y valores del que aprende y el cuestionamiento explícito puede llevar a una actitud crítica, a una consciencia cultural crítica" (Byram y Fleming, 2001, p. 6). Las distintas formas de ver el mundo, de acuerdo con las distintas culturas, permiten que los aprendientes de una segunda lengua interpreten e interrelacionen distintos sistemas culturales (Byram y Zarate, 1998), lo 
que puede además conducirlos a un mejor entendimiento de su propia cultura. Lo anterior evita el etnocentrismo (Derenowski, 2011) y favorece su desarrollo como hablantes multiculturales.

El aprendizaje de la cultura acelera la inmersión de los hablantes no nativos en la comunidad que los recibe, pues facilita el acceso a la realidad y a la actuación como agente social en distintas situaciones comunicativas determinadas por el contexto en que se produce el intercambio, por las condiciones sociales y mentales de los participantes, determinadas a su vez por referentes culturales comunes al grupo (Gago, 2010). El hecho comunicativo no puede comprenderse al margen del contexto y las circunstancias irrepetibles de la sociedad en que se produce. De igual forma, tampoco es posible concebir el aprendizaje de la lengua al margen de la realidad a la que esta pertenece, de la cual es resultado y herramienta de construcción social, tanto si hablamos de situaciones de inmersión como de no-inmersión (Gago, 2010). En relación con lo anterior, un estudio etnográfico Nguyen y Kellog (2010) revela cómo los aprendientes de LE que son involucrados en actividades sociales en las que se les da relevancia a sus posturas e identidades, cambian su entendimiento sobre los estereotipos y se transforman en agentes sociales positivos en nuevas comunidades de práctica.

\section{El papel de los profesores de LE}

De acuerdo con Kramsch (1996), el enseñanza-aprendizaje de la cultura les servirá a los aprendientes para la construcción de su competencia intercultural y de su identidad cultural propia, lo cual puede convertir a los profesores en agentes de cambio social. Ahora bien, el papel que asume un profesor puede estar influido por su cultura de origen. Al respecto, Can et al. (2011) advierten sobre la importancia de que los profesores presten atención a elementos contextuales o culturales. En su estudio, estos autores les pidieron a profesores de LE que completaran definiciones sobre su labor docente mediante metáforas. El análisis muestra cómo profesores polacos y turcos emplean metáforas diferentes para caracterizar su labor docente, muchas de las cuales pueden estar motivadas culturalmente.

Ante la diversidad cultural de nuestros aprendientes, su amplitud de intereses respecto a la adquisición de elementos culturales, la amplia gama de estilos cognitivos y la variedad de estilos de enseñanza que pueden estar determinados culturalmente, se plantea la pregunta respecto a qué enseñar para la adquisición del componente cultural en una lengua extranjera. En el transcurso del tiempo, los manuales de 
enseñanza a extranjeros han organizado la cultura tomando como referencia los parámetros de civilización, literatura, arte, geografía, historia y economía (Carcedo, 1998: 168). Esta diversidad impone que sea conocedor de todas estas áreas, lo que es una tarea difícil que, sin embargo, puede ser suplida por el conocimiento propio y la guía de nuestros propios aprendientes. Al respecto, es fundamental detectar los patrones culturales que deben impartirse en la clase, para lo cual Carcedo (1998) propone seguir una estrategia didáctica que implica (i) que nuestros propios alumnos sean nuestros informantes; (ii) que la clase sea el campo para la obtención de la información que necesitamos; y (iii) que las respuestas de nuestros alumnos conformen un corpus para la comparación y el análisis.

Otra solución al problema de qué enseñar tiene que ver con la interpretación correcta de los parámetros de la cultura y su integración en el aula bajo una comunicación intercultural (De Santiago, 2010). El proyecto de este autor Enciclopedia para la comunicación intercultural, se organiza a través de una encuesta sobre costumbres y hábitos sociales recogidas por el Marco común de referencia del Consejo de Europa (2002, p. 97-98): vida diaria; relaciones personales; valores, las creencias y actitudes; lenguaje corporal y comportamiento ritual. Nuestro trabajo tomará en cuenta estas opciones y otras investigaciones de autores del área de ELE, como las de Níkleva (2012), para cuyo estudio tomó como referencia las nueve categorías de Byram y Morgan (1994) y el Plan curricular... del Instituto Cervantes (2007), para desarrollar una clasificación de contenidos culturales en tres categorías: (i) referentes culturales; (ii) saberes y comportamientos socioculturales y (iii) habilidades y actitudes interculturales. Los referentes culturales se organizaron en: identidad; condiciones de vida y organización social; organización sociopolítica; interacción cultural y social (comportamientos socioculturales); historia y actualidad de los países hispanos; geografía de los países hispanos y herencia cultural.

Con escasas diferencias, los descriptores para la enseñanza del componente cultural coinciden entre los autores (Instituto Cervantes (2007); Navarro, 2009; Níkleva, 2012; Quiles y Cabrera, 2013). La última autora, no obstante, plantea contenidos que tienen que ver con lo que Navarro (2009) denominó "cultura enciclopédica" y que, como tal, es un área de contenido cuyo tratamiento se presta para el área de la educación superior o universitaria (Quiles y Cabrera, 2013, p. 205).

Para concluir este apartado sobre el papel del profesor en la enseñanza de la cultura nos remitiremos a Oliveras (2000), quien resalta varios principios imprescindibles: (i) la conservación de la identidad de los aprendientes, suavizando sentimientos nacionalistas a través de la reflexión; (ii) el papel del aprendizaje de la LE como parte 
del proceso de adquisición de la competencia intercultural; (iii) las implicaciones sociales de la educación intercultural: la necesidad de investigación y aprendizaje que incluya una evaluación crítica de los valores culturales establecidos y de los métodos tradicionales; (iv) la importancia de la observación etnográfica en la formación de profesores y en la confección de materiales didácticos; (v) el papel de los estudios de comparación entre culturas; y (vi) el hecho de que los debates internacionales deban considerar el contexto histórico-social al formular discursos de materia educativa o académica, para lo cual es fundamental conocer dichos contextos.

\section{Diseño y metodología}

Nuestro estudio se llevó a cabo en los cursos de español como lengua extranjera que imparte el Programa de Español de la Pontificia Universidad Católica de Chile (UC). La muestra seleccionada se compone de un número de 51 alumnos de diferentes niveles de lengua $\mathrm{A} 2-\mathrm{C} 1$, de acuerdo al Marco común europeo... del Consejo de Europa (2002). El diseño de la investigación consta de un cuestionario de veinte preguntas organizadas sobre dos ejes, que tratan sobre los referentes más seleccionados por parte de los estudiantes de ELE de la UC y los ítems culturales que más motivan al alumno a aprender la lengua española. Para el desarrollo de las respuestas se optó por una escala tipo Likert (1932), con el objeto de facilitar la cumplimentación de este instrumento.

El objetivo del diseño de la encuesta se basa en el análisis del conocimiento del alumno acerca de los referentes culturales del mundo chileno, considerando las nociones culturales que define el MCER mencionadas en la sección 6, y los elementos de la cultura presentes en el manual Punto C/ELE (Toledo, 2017, 2018; Bravo, 2018). Este manual se toma como guía para la selección de los referentes culturales porque es el recurso didáctico presente en las clases de español del Programa de Español de esta universidad.

En el diseño de la encuesta de escala tipo Likert se valora en base a una escala de cinco elementos configurados en dos secciones. El primer apartado mide el nivel de importancia de los referentes culturales asignado por los estudiantes, cuyo rango de valores es: muy importante, importante, moderadamente importante, de poca importancia y sin importancia. La segunda sección mide la valoración de los ítems culturales según experiencia y motivación, y para ello se presenta el rango de valores: totalmente de acuerdo, de acuerdo, ni acuerdo ni desacuerdo, en desacuerdo, totalmente en desacuerdo. 
El estudio organiza la encuesta en dos secciones: los referentes culturales más seleccionados, subdividido en diez apartados: literatura (a), cine (b), tratamiento específico de estereotipos (c), gastronomía (d), historia-política (e), movimientos sociales $(\mathrm{f})$, expresiones coloquiales $(\mathrm{g})$, música $(\mathrm{h})$, mundo indígena (i), tradiciones familiares (j). En la segunda sección, la encuesta mide el nivel de motivación e importancia que le aportan los ítems culturales a los aprendientes. Esta parte se subdivide en diez afirmaciones en positivo que relevan el factor motivacional en cada uno de los diez referentes culturales, por ejemplo:

- Descubrir los movimientos sociales y políticos de Chile (marchas) me motiva a aprender español.

- Las costumbres chilenas son un factor importante en mi proceso de aprendizaje.

Las afirmaciones se crearon pensando en el tratamiento del componente cultural que proponen los manuales PuntoCE/LE, el MCER y el PCIC, considerando contexto docente de la Universidad Católica de Chile.

El perfil del informante responde a un estudiante universitario de intercambio que toma cursos de diversas disciplinas en la UC (Ingeniería, Ciencias Políticas, Literatura, etc.) con un promedio de edad entre 20 y 24 años. Los informantes proceden de los Estados Unidos, Europa, Asia y Brasil. Se trata de universitarios, con un plan docente de intercambio de cuatro meses en la UC de Chile.

La selección de los referentes culturales de esta investigación se define sumando el inventario cultural del MCER con el manual Punto C/ELE (Toledo, 2017, 2018; Bravo, 2018). El cuadro 1 resume los referentes culturales extraídos de estas fuentes y que se consideraron en esta investigación.

Cuadro 1. Referentes culturales del mundo hispano analizados en la presente investigación
a) La literatura.
f) La política.
b) El cine.
g) Las expresiones coloquiales.
c) Los estereotipos.
h) La música.
d) La gastronomía.
i) El mundo indígena.
e) La historia.
j) Las tradiciones familiares.

La investigación de estos referentes culturales se presenta de dos maneras distintas: por medio de una escala de valoración respecto al tema cultural mismo que están aprendiendo a lo largo del curso de español y por el nivel de acuerdo o desacuerdo con distintas afirmaciones que hablan acerca de la motivación y significación que implican los diferentes ítems culturales. Con esta distribución se espera no solamente 
saber cuáles son los contenidos más interesantes de la culturales hispana para los estudiantes, sino también en dónde se implican más y en qué ítems su grado de motivación o interés aumenta.

A continuación, se analiza los resultados con respecto a los referentes culturales chilenos para definir el perfil cultural del alumno de español en la UC de Chile.

\section{Análisis y resultados}

El gráfico 1 ofrece una visión general de los diez ítems seleccionados en este estudio (en base al MCER y al manual Punto C/ELE) como nociones específicas en el aprendizaje intercultural del alumnado de español de la UC. Los referentes culturales se analizarán en dos sentidos: aquellos que están en tendencia alta y aquellos en tendencia baja según los resultados de las encuestas.

Cada uno de los contenidos son necesarios para el desarrollo de la competencia intercultural de un hablante de español como segunda lengua, sin embargo, los datos muestran que los aprendices tienen una visión diferente al respecto. En efecto, el gráfico 1 muestra que aspectos como la literatura, la gastronomía y la música tienen un valor menor de lo esperado. Esto es interesante dado que el tratamiento de estos temas en los manuales de enseñanza de EE es bastante frecuente.

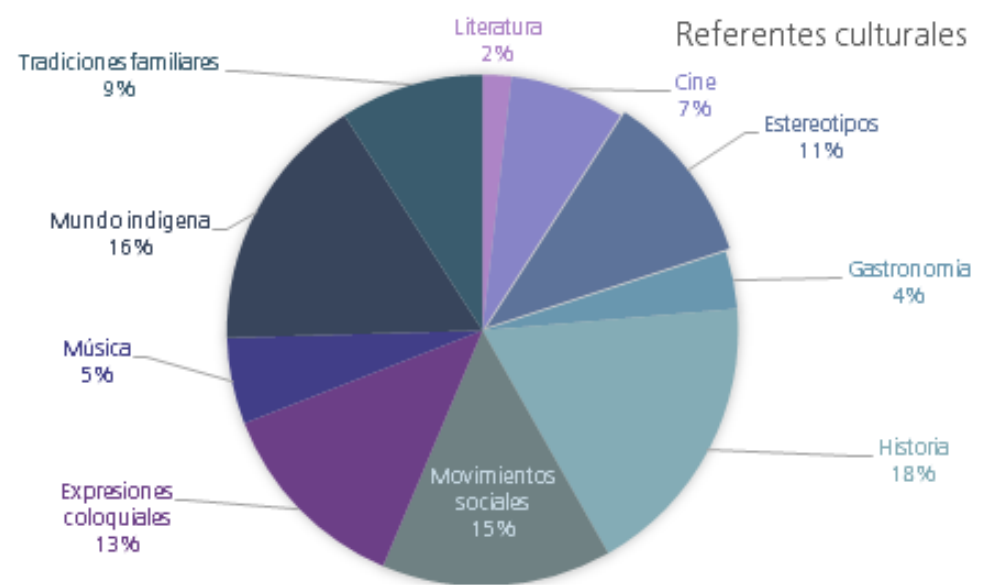

Gráfico 1. Preferencias del alumnado por los distintos elementos culturales. 
Los ítems ilustrados en el gráfico 2 ponen de manifiesto un fuerte aumento de los referentes culturales que mantienen una vinculación con el contexto social. La historia, el mundo indígena, los movimientos sociales, las expresiones coloquiales, los estereotipos y las tradiciones familiares (en orden de preferencia). La selección de las expresiones coloquiales y los estereotipos reflejan un perfil de aprendiz centrado en las tendencias actuales y en la cultura popular. Hay que tener en cuenta que son alumnos en inmersión que buscan responder sus necesidades comunicativas puntuales y estos referentes culturales pueden ayudarles a dibujar una imagen sobre el país extranjero en el que se encuentran inmersos.

Referentes culturales con mayor nivel de interés del alumnado
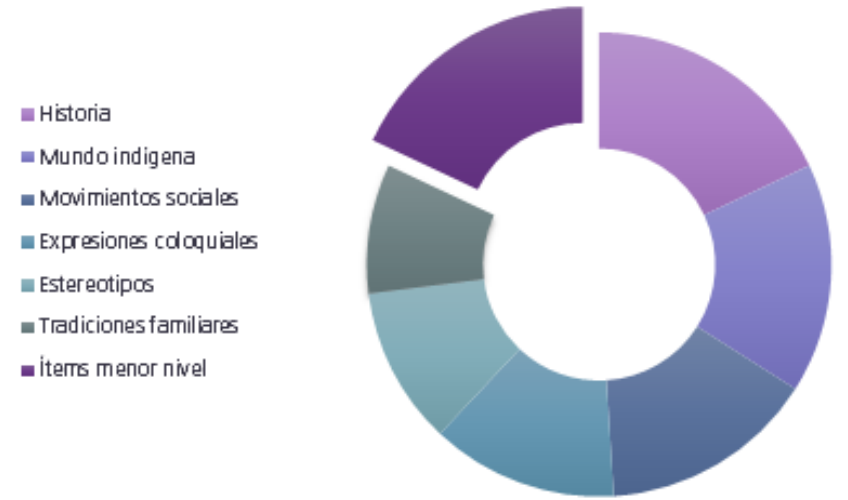

Gráfico 2. Referentes culturales con mayor nivel de interés del alumnado.

La interpretación del gráfico 2 plantea un primer desafío para el docente, quien tendrá que responder a la tensión entre las llamadas cultura con mayúscula y la cultura con minúscula (Miquel y Sans, 1992). Conforme a estas tendencias, las clases de español tienen que orientarse hacia temáticas como son la herencia de la cultura chilena y el movimiento político actual del país, pues estos los contenidos culturales que generan interés en el proceso de aprendizaje del alumnado. De acuerdo con esto, se concibe un perfil de alumno implicado socialmente en el país donde está inmerso lingüísticamente. Gracias a la vivencia de la cultura, estos alumnos muestran preocupación por las costumbres y el contacto con la sociedad chilena, lo que los hace interesarse por las expresiones coloquiales y los estereotipos del nativo. 
El gráfico 3 expone aquellos ítems que muestran una menor frecuencia de selección, a pesar de tener una posición prevalente en los manuales de ELE, porque se relacionan con la cultura enciclopédica (Navarro, 2009): la literatura, la música, el cine o la gastronomía, que son referentes culturales tradicionalmente seleccionados por los editores de los manuales de la enseñanza de ELE.

\section{Referentes culturales con menor nivel de interés del alumnado}
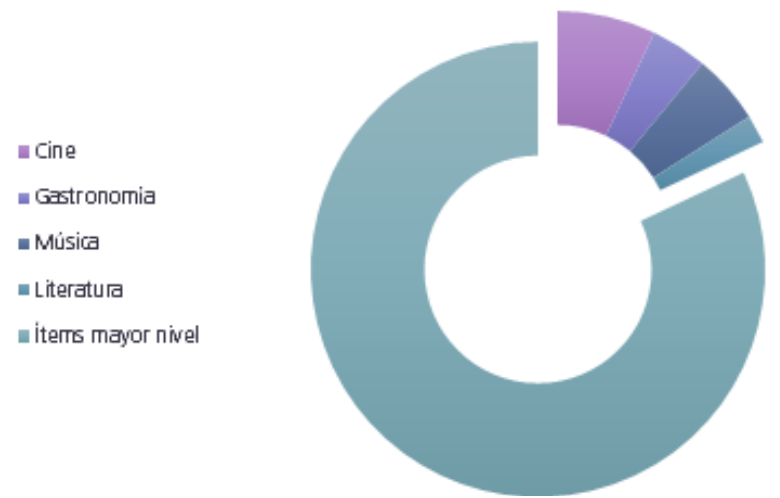

Gráfico 3. Referentes culturales con menor nivel de interés del alumnado.

Los alumnos relacionan estos elementos con información que pueden adquirir en los libros de ELE. La cultura cotidiana, en cambio, únicamente puede adquirirse o experimentarse $y$, en ese sentido, la inmersión ofrece una oportunidad única de encuentro intercultural auténtico. Los datos que muestra el gráfico 3 nos llevan a cuestionar si acaso los aspectos culturales relativos a la cultura enciclopédica son realmente los más propicios para motivar la enseñanza-aprendizaje de ELE. Al parecer, el estudiante de español de la UC atribuye un mayor valor a la experiencia social que le aporta el conocimiento de la historia y la cultura popular.

Otro aspecto que se investiga en este trabajo es el nivel de motivación del alumnado de español acerca de una serie de afirmaciones relacionadas con los referentes culturales. El objetivo es la reflexión y la opinión crítica del estudiante sobre su aprendizaje cultural. A continuación, los gráficos 4 y 5 muestran la orientación sobre los ítems culturales que los alumnos consideran más significativos en su aprendizaje. Estos gráficos evalúan los referentes culturales según el nivel motivacional del estudiante: el gráfico 4 muestra los ítems culturales "enciclopédicos" en los que destaca el cine, la música, la literatura y la gastronomía, y el gráfico 5 expone los referentes culturales "experienciales" donde se encuentran las tradiciones familiares, las expresiones coloquiales, el indigenismo chileno y los movimientos sociales del país. 
En cuanto a la consideración motivacional de los alumnos acerca de los referentes culturales, el gráfico muestra con valores negativos y positivos una visión general del perfil experiencial del estudiante de español en Chile que se ha dibujado a lo largo del estudio. Como puede apreciarse en el gráfico 4, los referentes culturales como el cine, la música o la literatura son valorados positivamente. Esta paradoja respecto a los resultados de Preferencias del alumnado por los distintos elementos culturales, puede deberse a que los estudiantes no relacionan cada elemento con un ítem específico de la cultura enciclopédica chilena, sino que entregan su opinión considerando la motivación asociada a estos.

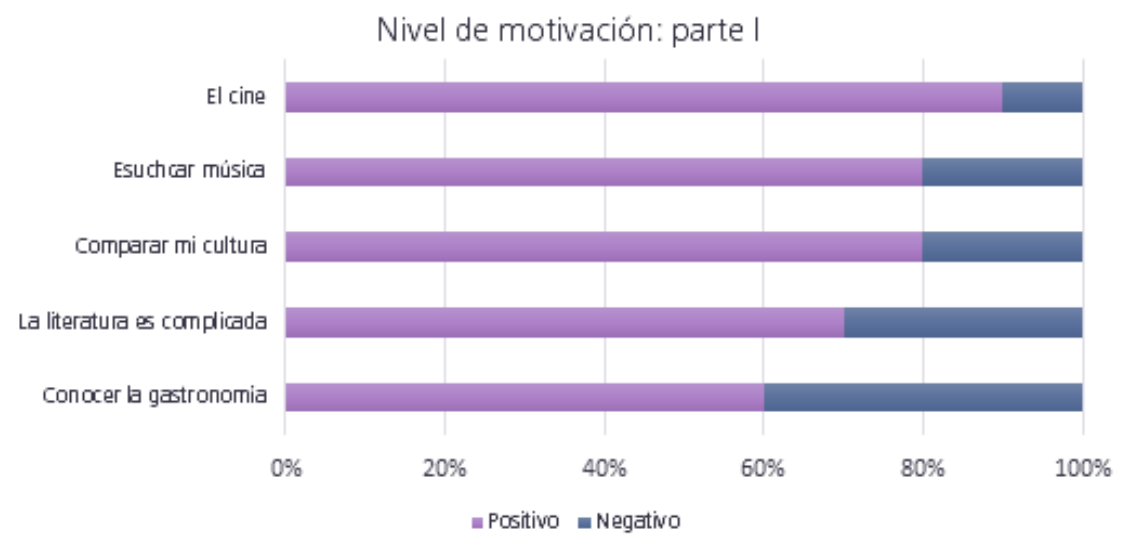

Gráfico 4. Nivel de motivación e importancia (parte I) del alumnado.

En el gráfico 4 se consideraron los valores entre 1 y 2 de la escala de Likert como positivos, y los valores 3,4 y 5 como negativos. De esta forma se crearon dos grupos para medir y representar en el gráfico la motivación con valor afirmativo o negativo. Lo que se observa, es un incremento positivo del cine cuando aparece como un juicio personal donde el alumno valora: "el cine es un referente cultural que me ayuda a aprender mejor español" (Anexo). A partir de las anotaciones que varios informantes incluyeron en sus cuestionarios, pudimos observar que los alumnos piensan que el cine ayuda cuando aprenden una segunda lengua porque puede reconocer las diferentes variedades de la lengua española como el chileno, el mexicano, el español de España, etc. De la misma manera ocurre con la música, los aprendices piensan que su aprendizaje puede mejorar gracias a los beneficios que puede aportar la música, pero que los artistas hispanos que conocen son "muy conocidos" y que les gustaría descubrir a cantantes chilenos porque desconocen artistas musicales del país. 
La literatura y la gastronomía muestran un incremento gradual en su valoración, posicionándose como dos referentes culturales que según los datos plantean un nuevo paradigma. En un primer momento, el dato referente a la gastronomía puede asociarse al conocimiento "experiencial" de la cultura, por el que mostraron preferencias antes. Por otra parte, la literatura (70\%) es un contenido que al alumno le resulta complicado comprender según su nivel y que puede llegar a percibir como un contenido menos experiencial que otros referentes culturales. Como resultado, todos estos datos muestran una preferencia en el alumnado más hacia lo visual o lo musical (no necesariamente artístico) que a la lectura. Esto plantea un desafío al docente, que tendrá que identificar, seleccionar y evaluar la literatura más cercana al alumnado, para no dejar de promover la destreza lectora en el aprendizaje de español. Podría afirmarse que, en este segundo momento, nuestro estudio revela un alumno de español que vincula su aprendizaje a los aspectos visual y social, como consecuencia de esto, la gastronomía no alcanza tanta atención como el cine o la música.

A continuación, en el gráfico 5 se mide el grado de opinión sobre aquellos referentes que tratan contenidos socioculturales. Los datos muestran un patrón donde la cultura y la tradición popular son aspectos motivacionales en el proceso de aprendizaje del alumno de ELE de la UC.

Los referentes culturales que según nuestros informantes los motivan para aprender la lengua española son los ítems: "el conocimiento de las costumbres chilenas es importantes en mi aprendizaje y comprender chilenismos resulta útil en mi proceso de aprendizaje". Por lo visto, estos aprendientes perciben una oportunidad de aprendizaje más informal y motivacional en la cultura popular y coloquial chilena, que puede acercarlos a su propia identidad sociocultural.

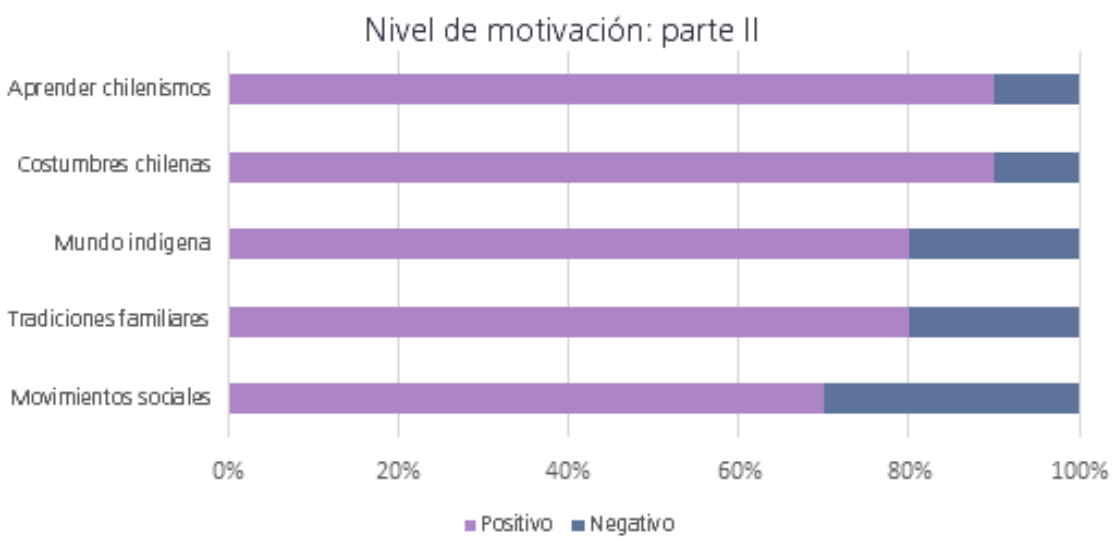

Gráfico 5. Nivel de motivación e importancia (parte II) del alumnado. 
Comparando los datos de los gráficos 2 y 5 se comprueba un constante aumento en el ítem relacionado con las costumbres chilenas, debido al interés del alumno por la tradición indígena, las rutinas y las costumbres propias del país. Este resultado concuerda con las selecciones que discutimos en el apartado $n^{\circ} 2$, de acuerdo con lo cual los aprendientes de la UC se acercan más a un perfil "experiencial" de la cultura. Las selecciones de estos alumnos nos parecen positivas para su proceso de adquisición y desarrollo del español chileno y de su identidad intercultural. Este patrón de estudiantes, con un alto interés en experimentar la cultura, los aleja de falsos estereotipos y los pone en contacto continuo con la sociedad meta, la cual a su vez los orienta en un contexto real sobre el componente sociocultural local.

Los datos observados en el aprendizaje de chilenismos sugieren la importancia que le da el alumno al léxico coloquial y las oportunidades que le aporta el registro informal como hablante en una situación de inmersión lingüística. La capacidad de reconocer, comprender y utilizar expresiones propias de la segunda lengua los hace sentir más cercanos a los hablantes nativos. Además, teniendo en cuenta el carácter experiencial-social de este alumnado, es normal que tiendan a un registro coloquial con la finalidad de entablar relaciones con la nueva cultura, sin embargo, la tendencia hacia lo coloquial puede generar que el alumno asocie por error el registro informal solamente a la oralidad. En este sentido, la función del docente es relacionar en clase este registro tanto con la escritura como con la oralidad y promover un tipo de literatura como el texto periodístico, el teatro o incluso el género de la publicidad, que ayude a captar el interés del alumno por el referente cultural literario.

Los resultados del ítem acerca de las tradiciones familiares parecen confirmar la vinculación social en el proceso de enseñanza-aprendizaje de nuestros informantes. El enunciado "conocer las tradiciones familiares chilenas me ayudará a comprender mejor su cultura" aparece con una evaluación positiva del estudiante de español que llega a la UC. Datos como este ponen de manifiesto un alto grado de sensibilización por la cultura popular, que es la que se produce en el día a día y en relación con los nativos de la nueva cultura. Es importante señalar que la mayoría de los estudiantes de español de la UC comparten con familias chilenas, por lo cual aprenden no solo la lengua, sino la cultura desde todas las perspectivas posibles.

El último referente cultural dentro del apartado de grado de motivación hace referencia a los movimientos sociales-políticos (marchas, manifestaciones) de Chile. En esta área se muestra un patrón comprometido y colaborador en la cultura chilena. Es interesante comprobar que, solamente durante un cuatrimestre, este grupo de- 
sarrolla una fuerte empatía respecto a los movimientos involucrados en la situación sociopolítica local. Esta actitud enriquece su competencia sociocultural y ayuda al docente a crear distintas oportunidades y estrategias de aprendizaje intercultural. En las encuestas se observa que a los informantes les interesaría saber más sobre aquellas huelgas que afectan a los jóvenes, pues esto los haría más partícipes en la sociedad chilena.

Un punto a analizar es por qué este referente obtuvo menor evaluación en esta encuesta, mientras que en la primera encuesta fue la segunda opción más valorizada. Pareciera ser que los aprendientes se sienten más motivados por elementos de la cultura popular, sin embargo, el hecho de no sentir una motivación tan fuerte por el tema de los movimientos sociales, no les impide reconocer su importancia.

\section{Conclusiones y futuras líneas de investigación}

En este estudio se ha analizado el papel de los referentes culturales chilenos en los cursos de español de la UC y se ha explicado la importancia de la actualización de los ítems culturales según el nivel motivacional en el contexto de aprendizaje de ELE. Los puntos principales de este trabajo han sido el análisis de la situación actual de la enseñanza de español en Chile y la evaluación de los referentes culturales chilenos mediante encuestas que medían la escala de frecuencia de selección de contenidos que motivan al alumno a aprender la lengua.

En esta investigación se evaluaron los ítems culturales enmarcados en los cursos de español de la UC con el fin de conocer las apreciaciones de los aprendientes de ELE y orientar hacia una mejora sobre la selección de los contenidos culturales en Chile. Los datos se obtuvieron mediante el diseño de una encuesta tipo Likert dividida en dos ejes que por un lado medían el nivel de frecuencia de selección de diez ítems que desean aprender y, por otro lado, el nivel de motivación de diez categorías. Las respuestas "totalmente de acuerdo y de acuerdo" se agruparon en un polo positivo y las respuestas con valor "ni acuerdo ni desacuerdo, en desacuerdo y totalmente en desacuerdo" se agruparon en uno negativo. Los informantes mostraron especialmente respuestas con valores en extremos (totalmente de acuerdo o totalmente en desacuerdo), lo que nos permitió tratar el valor de las respuestas de esta forma. El grupo de informantes fue compuesto por 51 estudiantes universitarios procedentes de Estados Unidos, Europa, Asia y Brasil que en ese momento aprendían mediante inmersión lingüística la lengua española en la UC durante un periodo de cuatro meses. 
Este estudio ha demostrado que, por un lado, los referentes culturales no pueden ser planteados de una forma universal, sino que tienen que ser seleccionados según el contexto de enseñanza-aprendizaje. Este hecho sugiere que el planteamiento de un inventario cultural en ELE tiene que estar actualizado según la variante de la lengua española que enseñen, los referentes teóricos que utilicen como manual y el nivel de motivación del alumnado hacia los ítems culturales. Por otro lado, el segundo hallazgo fue la falta de correspondencia entre la clasificación cultural tradicional de los editores en los manuales de ELE y el criterio del estudiante. De cualquier forma, creemos que una futura investigación podría esclarecer, mediante entrevistas, si los contenidos de los manuales tradicionales de ELE cubren realmente todos los intereses de los aprendientes. Lo que parece muy claro es que el estudio del componente cultural es un proceso en constante cambio y por esta razón, la evaluación continua de los referentes culturales es una técnica importante para la enseñanza de ELE.

Los resultados de esta investigación apoyan la idea de Navarro (2009) que divide la cultura en tres niveles: enciclopédica, epidérmica y pragmática. Los datos indican que los estudiantes asocian la literatura, el cine, la gastronomía y la música a lo que tradicionalmente se define como cultura legitimada. De esta manera, el nivel motivacional es débil porque estos referentes son asociados a la información recogida en los libros o a los contenidos relacionados a la cultura con mayúsculas (Miquel y Sans, 1992). Por otra parte, los ítems culturales asociados a lo experiencial como son la tradición, las marchas políticas, el indigenismo chileno y las expresiones coloquiales manifiestan un nivel motivacional elevado. Estos resultados demuestran que el perfil del estudiante de ELE en las clases chilenas se define por lo experiencial, la observación y el empirismo.

Aunque el estudio ha demostrado los referentes culturales más frecuentes según el perfil experiencial del estudiante de ELE en Chile, estos resultados podrían no ser aplicables a los demás cursos de español impartidos en el país. Esta investigación se diseñó específicamente para evaluar los factores relacionados con la cultura chilena en los cursos de español de la UC. Sin embargo, el hecho de haber diseñado una encuesta según los referentes culturales que están presentes en el único manual de ELE en Chile da la posibilidad de aplicarla en un futuro a las otras instituciones que imparten ELE. De esta forma se podría obtener una visión total de los referentes culturales chilenos y proponer una orientación sobre la enseñanza de la cultura en este país. 
Los resultados de este estudio proporcionan una contribución para el entendimiento del campo de ELE en Chile, ya que establece una aproximación del panorama actual de la enseñanza de español en dicho país y contribuye en la evaluación de los contenidos culturales de ELE con el fin de orientar sobre su enseñanza. Esta aproximación ha permitido realizar una mejor explicación de aquellos referentes que necesitan ser actualizados según el nivel de frecuencia y motivación del alumnado de español en este país. El perfil que estudia español en la UC busca un aprendizaje experiencial ligado a los referentes significativamente prácticos como son las marchas políticas, las expresiones coloquiales o las tradiciones familiares. Estos datos subrayan la importancia de los factores motivacionales en la enseñanza de SL para desarrollar un aprendizaje significativo en el estudiante.

De este trabajo surge una nueva e interesante observación en el planteamiento de los referentes culturales para el desarrollo de un aprendizaje significativo. Como los datos muestran una tendencia hacia lo experiencial, las clases podrían plantear tareas didácticas donde los estudiantes intercambiaran información necesaria con nativos chilenos. De esta forma, los referentes culturales que reciben menor atención se manifestarían de una forma práctica: una primera propuesta didáctica sería el festival de cine chileno desde una perspectiva interdisciplinar. De esta forma el cine se aprendería mediante la historia, la geografía, el arte, la música, entre otras disciplinas. Además, se plantearía la creación de un proyecto audiovisual personal en español con la participación de nativos chilenos. Estas tareas, que suponen una implicación tanto lingüística como emocional, producen un aprendizaje significativo en el estudiante. La segunda propuesta didáctica sería el día internacional de la comida donde nativos y extranjeros comparten un plato típico y las tradiciones que subyacen en la elaboración de esa receta. Los estudiantes aprenden conocimientos experienciales de la gastronomía mediante preguntas a los nativos y luego se les plantea un proyecto según las respuestas que hayan obtenido. La última propuesta didáctica que denominamos El festival del libro implicaría la narración de historias, que tendría como temática los referentes culturales y los mitos literarios chilenos. La técnica de narración de historias motivaría al alumno a aprender la literatura de una manera original, creativa y significativa para su aprendizaje de ELE.

Los resultados de este estudio proporcionan las siguientes ideas para futuras investigaciones en el campo de ELE. Por un lado, el análisis de los referentes culturales en las distintas instituciones que imparten cursos de español para comparar y plantear un inventario adaptado al contexto cultural local. La idea de una clasificación cultural definida en términos de nivel de frecuencia ayudaría en el diseño de materiales de ELE y ofrecería una visión de las semejanzas y diferencias con respecto a otros países. 
Por otro lado, la investigación de las estrategias y las técnicas de aprendizaje de los estudiantes en la adquisición del componente cultural. Sería interesante una línea de investigación acerca del procedimiento de aprendizaje cultural del alumno de ELE local, cuáles son las estrategias de enseñanza-aprendizaje que integra, de qué manera las incorpora y cómo lleva a cabo la organización de esas estrategias en su aprendizaje. El reconocimiento de las diferentes formas de aprendizaje de los referentes culturales podría establecer categorías de estilos de aprendizaje en los estudiantes de ELE. Esta línea de investigación sería positiva para establecer teorías de aprendizaje en el área de ELE y para demostrar cómo los alumnos aprenden el componente cultural en términos de afectividad y de motivación.

\section{Referencias bibliográficas}

Akinyemi, A. (2005). Integrating Culture and Second Language Teaching through Yorubá Personal Names. The Modern Language Journal, 89(1), 115 - 126.

Areizaga, E. (2001). Cultura para la formación de la competencia comunicativa intercultural: el enfoque formativo. Revista de Psicolingüística, 12, 157-170. https://bit. ly/2OsKOcg

Bravo, D. (2018). Manual PuntoCE/LE. Nivel B2. Santiago: Ediciones Pontificia Universidad Católica de Chile.

Byram, M. \& Morgan, C. (1994). Teaching-and-Learning language and culture. Clevedon: Multilingual Matters.

Byram, M. y Fleming, M. (2001). Perspectivas interculturales en el aprendizaje de idiomas. Madrid: Cambridge University Press.

Byram, M. y Zarate, G. (1998). Definitions, objectives and assessment of sociocultural competence. En Byram, M., Zarate, G. y Neuner, G. (eds.). Sociocultural Competence in language learning and teaching. Strasbourg: Council of Europe.

Can, C., Bedir, H. y Kiliánska-Przybylo (2011). Is Teaching Culture-Bound? A Cross-Cultural Study on the Beliefs of ELT teachers. En Pawlak, M. (Ed.) Aspects of Culture in Second Language Acquisition and Foreign Language Learning (pp. 107-120). Silesia, Poland: Springer.

Canale, M. y Swain, M. (1980). Theoretical bases of communicative approaches to second language teaching and testing, Applied Linguistics, 1, 1-47.

Carcedo, A. (1998). Cultura y patrones de comportamiento: su integración en la enseñanza de la lengua. En Celis A. y Heredia J. R. (Coords. ed.), Lengua y cultura en la enseñanza de español a extranjeros. Actas del VII Congreso Internacional de ASELE, pp. 165-173. Cuenca: Ediciones de la Universidad de Castilla - La Mancha. 
Consejo de Europa (2002). Marco común europeo de referencia para las lenguas: aprendizaje, enseñanza, evaluación. Madrid: Anaya.

Corros, F.J. (2008). La evaluación de la competencia cultural. XVIII Congreso Internacional de la Asociación para la Enseñanza del Español como lengua Extranjera (ASELE): 19-22 de septiembre de 2007 / coord. por Susana Pastor Cesteros, Santiago Roca Marín. pp. 234-239. Alicante. https://bit.ly/2YmjvVK

De Santiago. J. (2010). La competencia cultural en la competencia comunicativa: hacia una comunicación intercultural con menos interferencias. MarcoELE, Revista de Didáctica Español Lengua Extranjera, 11, 113-130. https://bit.ly/2ZsGqzO

Denis, M. y Matas ,M. (2009). Para una didáctica del componente cultural en clase de E/LE. MarcoELE, 9, 87-95. https://bit.ly/2tOa6c4

Derenowski, M. (2011). Strangers in Paradise: The Role of Target Language Culture in Foreign Language Teaching Materials. En Pawlak, M. (Ed.) Aspects of Culture in Second Language Acquisition and Foreign Language Learning. (pp. 273-286) Silesia, Poland: Springer .

Díaz, H. (2006). El componente cultural en la enseñanza de una lengua extranjera como aplicación didáctica, Aldadis.net La revista de educación, 9, 9-12. https://bit. ly/2SKutmC

Ferreira, A. (2017). Panorama del español como lengua extranjera en Chile. ASELE. Fundación San Millán de la Cogolla. https://bit.ly/2SN10J2

Gago, E. (2010). El desarrollo de I competencia intercultural en el aula de ELE. Analecta Malacitana (AnMal electrónica), 29, 221-239. https://bit.ly/2ZifhQj

García, P. (2004). La cultura, ¿universo compartido? La didáctica intercultural en la enseñanza de idiomas. RedELE: Revista electrónica de Didáctica ELE, 1-6.

Grupo CRIT (2006). Culturas cara a cara. Relatos y actividades para la comunicación intercultural. Madrid, Edinumen.

Hymes, D.H. (1972). On Communicative Competence. In: J.B. Pride \& J. Holmes (eds). Sociolinguistics. Selected Readings. Harmondsworth: Penguin.

Instituto Cervantes (2009). CVC. Diccionario de términos clave de ELE [en línea]. https:// bit.ly/1wSua5p

Instituto Cervantes. (2007). Plan Curricular del Instituto Cervantes. Madrid: Biblioteca Nueva.

Kramsch, C. (1995). The cultural component of language teaching. Language, culture and curriculum, 8(2), 83-92.

Lee, E. (2015). Doing culture, of doing race: everyday discourses of 'culture'and 'cultural difference' in the English as second language classroom, Journal of Multilingual and Multicultural Development, 36(1), 80-93.

Likert, R. (1932). A technique for the measurement of attitudes. Archives of Psychology, $22,140$. 
Miquel, L. y Sans, N. (1992). “El componente cultural: un ingrediente más de la clases de lengua", en Cable, 9.

Navarro, P. (2009). Cultura con eñe: cultura, sociocultura e intercultura en la clase de ELE, TINKUY: Boletín de investigación y debate, 11, 83-93. https://bit.ly/2GMYfTh

Nguyen, H. T. y Kellog, G. (2010). I Had a Stereotype That American Were Fat: Becoming a Speaker of Culture in a Second Language, The Modern Language Journal, 94(1), 56-73.

Níkleva, G. (2012). La competencia intercultural y el tratamiento de contenidos culturales en manuales de español como lengua extranjera. RESLA, 25, 165-187. https:// bit.ly/2YtkNSW

Oliveras, A. (2000). Hacia la competencia intercultural en el aprendizaje de una lengua extranjera. Estudio del choque cultural y los malentendidos, Barcelona-Madrid, Universitat de Barcelona: Edinumen.

Otwinowska-Kastztelanic, A. (2011). Do We Need to Teach Culture and How Much Culture Do We Need? En M. Pawlak (Ed.) Aspects of Culture in Second Language Acquisition and Foreign Language Learning (pp. 35-48). Silesia, Poland: Springer.

Quiles, M. y Cabrera, M. P. (2013). Sobre el texto y la competencia cultural en la Educación Superior: aproximación a un aula de ELE. Porta Linguarium, 199-217. https:// bit.ly/2Yp4l6h

Quirós, G. (2018). La docencia de ELE en Chile y el uso de manuales extranjeros en el aula. MarcoElE: Revista de Didáctica Español Lengua Extranjera, 26.

Sapir, E. (1954). El lenguaje, México DF, México: Fondo de Cultura Económica.

Toledo, G. (2017). Manual PuntoCE/LE. Nivel B1. Santiago: Ediciones Pontificia Universidad Católica de Chile.

Toledo, G. (2018). Manual PuntoCE/LE. Nivel A1. Santiago: Ediciones Pontificia Universidad Católica de Chile.

Viñó, G. M. y Massó, P. A. (2015). ¿Qué cultura deberíamos enseñar en el aula de ELE? Actas de I Congreso de español como lengua extranjera del Magreb. Instituto Cervantes 


\section{Anexo}

\begin{tabular}{|l|c|c|c|c|c|}
\hline & $\begin{array}{c}\text { Total- } \\
\text { mente de } \\
\text { acuerdo }\end{array}$ & $\begin{array}{c}\text { De } \\
\text { acuer- } \\
\text { do }\end{array}$ & $\begin{array}{c}\text { Ni acuerdo } \\
\text { ni des- } \\
\text { acuerdo }\end{array}$ & $\begin{array}{c}\text { En des- } \\
\text { acuerdo }\end{array}$ & $\begin{array}{c}\text { Totalmente } \\
\text { en des- } \\
\text { acuerdo }\end{array}$ \\
\hline $\begin{array}{l}\text { Comparar mi cultura con la cultura } \\
\text { chilena es importante en mi aprendizaje. }\end{array}$ & 1 & 2 & 3 & 4 & 5 \\
\hline $\begin{array}{l}\text { Las costumbres chilenas son un } \\
\text { factor importante en mi proceso de } \\
\text { aprendizaje. }\end{array}$ & 1 & 2 & 3 & 4 & 5 \\
\hline $\begin{array}{l}\text { El cine es un referente cultural que } \\
\text { me ayuda a aprender mejor español. }\end{array}$ & 1 & 2 & 3 & 4 & 5 \\
\hline $\begin{array}{l}\text { Descubrir los movimientos sociales } \\
\text { y políticos de Chile (marchas) me } \\
\text { motiva a aprender español. }\end{array}$ & 1 & 2 & 3 & 4 & 5 \\
\hline $\begin{array}{l}\text { Aprender expresiones coloquiales 0 } \\
\text { chilenismos es útil y motivador para } \\
\text { aprender español. }\end{array}$ & 1 & 2 & 3 & 4 & 5 \\
\hline $\begin{array}{l}\text { Conocer la gastronomía chilena es } \\
\text { un referente cultural que me motiva } \\
\text { para aprender más. }\end{array}$ & 1 & 2 & 3 & 4 & 5 \\
\hline $\begin{array}{l}\text { La literatura es un referente } \\
\text { complicado para mi nivel de } \\
\text { español, pero me motiva aprender } \\
\text { con obras literarias. }\end{array}$ & 1 & 2 & 3 & 4 & 5 \\
\hline $\begin{array}{l}\text { Conocer las tradiciones familiares } \\
\text { de los chilenos me ayudará a } \\
\text { comprender mejor su cultura. }\end{array}$ & 1 & 2 & 3 & 4 & 5 \\
\hline $\begin{array}{l}\text { Comprender la cultura indígena me } \\
\text { parece interesante y enriquecedor en } \\
\text { mi aprendizaje. }\end{array}$ & 1 & 2 & 3 & 4 & 5 \\
\hline $\begin{array}{l}\text { Escuchar música y conocer nuevos } \\
\text { grupos musicales en español mejora } \\
\text { mi aprendizaje. }\end{array}$ & 1 & 2 & 3 & 4 & 5 \\
\hline
\end{tabular}

